\title{
El fin de la ALFIN
}

\section{Antonio Calderón-Rehecho*}

Alfons Cornella, quien acuñara el afortunado término de infoxicación, reflexiona en el prólogo de Más allá de Google (Fernández García, 2008, pp. 8-9) sobre qué es la información ("esa sustancia integral de nuestro hoy"), de la que probablemente apenas sabemos nada porque pertenecemos a la generación que la ha inyectado en el motor del mundo (inmigrantes"), o porque la experimentamos desde el contexto de su uso, formando parte de nuestra vida (sólo es tecnología para los que nacieron antes que ella. Dentro de unas generaciones "quizá se dirá que el universo existe porque la información se hizo tiempo"). Porque es omnipresente y aumenta sin cesar, haciéndose inabarcable hasta el punto que si estableciéramos comparaciones a escala individual podríamos decir que la información de un único dominical del New York Times en 1997 contenía más información de la que estaba al alcance de la vida de un habitante europeo "medio" del siglo xviI (Ramonet, 2011, p. 23) y si lo hiciéramos a nivel global, cómo en los últimos cinco años se ha creado más información que en los 2.000 años anteriores $^{1}$. Es el resultado no sólo de la actividad de las tradicionales fuentes de información; también de la creación y la reutilización que el usuario conectado a Internet realiza (se trata de un número enorme de personas: más de $2.000 \mathrm{mi}-$ llones; pero todavía lejos de los 7.000 millones que pueblan el planeta) y de las estelas que dejamos a nuestro paso por las redes, que son el fundamento de negocios presentes y futuros, conformando lo que se concibe ya con el término de Big data, cercano también a la Internet de las cosas, que acabará por sumarse a la ingente masa de información, cada vez disponible en más lugares, creada con más aplicaciones, utilizadas desde más dispositivos, artilugios, máquinas... y -paradójicamente, a pesar de la existencia de open data- cada vez en menos manos, las de aquellos que son capaces precisamente de fiscalizar nuestros pasos en el maremagnum de la red y las que concentran los medios de comunicación de masas, ligados a grandes grupos industriales financieros. Este exceso de infor-

* Biblioteca de la Universidad Complutense de Madrid.

Correo-e: acalderon@buc.ucm.es

1 Entre 1967 y 1997 más que en los 5.000 anteriores (Ramonet, 2011, p. 23). 
mación es además, en muchos contextos y situaciones, totalmente deliberado, al mismo tiempo que premeditadamente equívoca o confusa la información (Otte, 2011) de tal manera que quien deba utilizarla vea disminuida su capacidad crítica ante la toma de decisiones en la vida cotidiana.

Será, por tanto, preciso conocer cómo desmantelar estos engaños, cómo usar esos artilugios, cómo obtenerlos, cómo sacar partido a aquellas aplicaciones y ser capaz de detectar -y adentrarse en- los lugares donde reside la información, cada vez más lejanos de la idea original de Internet como un espacio no acotado y sin vallados, donde los aspectos legales están en permanente discusión porque han cambiado buena parte de los modelos de negocio, de los actores y de la manera de establecer los flujos de relación e intercambio. Todo esto consiguiendo mantener una identidad digital que encuentre respeto a la privacidad y la confidencialidad. Es decir, tendremos que acostumbrarnos a aprender de manera constante y reconvertirnos. Da igual qué rostro tome o en qué formato pretendan hacernos morder la manzana del nuevo paraíso, no se tratará sino de información, lo que el ser humano utiliza para comunicarse y crear conocimiento, belleza, placer, bienestar... También desigualdad y sometimiento.

Aunque el usuario se sienta muy seguro moviéndose en este mundo que mencionamos, la realidad es que se encuentra si no desvalido sí abrumado por la ingente cantidad de recursos a los que se enfrenta si quiere resolver una necesidad informativa de cierta complejidad. Afecta a todos los aspectos de la vida: el personal, el ocio, el trabajo, el estudio, la enseñanza, el aprendizaje, la investigación... Por eso es por lo que además de desarrollar herramientas y técnicas para buscar y filtrar la información de manera automática, o casi, también se ha desarrollado en el mundo de los que trabajan con la información estrategias, programas, iniciativas, modelos, marcos, normas, planes... que buscan ayudar a los demás, aparte de a sí mismos, a poder maniobrar, a gestionar los recursos y obtener beneficios y satisfacciones de ese mundo infoxicado. No es sino la construcción de una identidad digital (porque el principal soporte de la información ahora es digital) consciente de los diferentes papeles que juega (el privado/público, el profesional/personal, el individual/colectivo, el productor/consumidor: Lara, 2010), de un entorno de aprendizaje personal y colectivo, en un mundo en el que la información es ubicua, multiplataforma, soportada sobre todo en hipermedia, con unos objetivos que no siempre se expresan directamente e interrelacionando personas que interpretan diferentes roles, desde ecosistemas digitales dispares, si no contrapuestos entre sí. Es el fin último de la ALFIN. Area y Pessoa (2012) afirman que "ser alfabeto es construirse una identidad digital como ciudadano autónomo, culto y con valores democráticos".

No se consigue en soledad, sino a través de un verdadero trabajo en equipo, interdisciplinar, con personas de distintos perfiles y procedencias, resolviendo problemas reales, tomando como partida cualquier elemento que pueda ser útil (Gómez-Hernández, 2012, pone como ejemplo el cómic), contextualizando respecto a temas, niveles y tipos de usuarios (por ejemplo, los proyectos docu- 
mentales integrados que diseña Rosa Piquín, (CEIP, 2011)), como único medio de conseguir interiorizar la información, haciéndola conocimiento.

La pujanza de tales iniciativas es visible. Valgan cuatro ejemplos documentales en nuestro país:

- la creación y desarrollo del portal ALFARED (2008), que engloba recursos ALFIN sin importar los ámbitos, recoge las aportaciones de un blog anterior creado por José Antonio Gómez-Hernández y se asienta sobre un Grupo de Trabajo del Consejo de Cooperación Bibliotecaria: GTALFIN;

- la del de CI2 (2011), fruto de la labor del grupo de trabajo que engloba dentro de la CRUE a su Comisión Sectorial sobre TIC con REBIUN, centrado - por su propio origen- en bibliotecas universitarias y abarcando competencias en información y competencias tecnológicas;

- el monográfico previsto para la desaparecida Educación y Biblioteca y que ha sido acogido en el seno de ALFARED (Veintitantas experiencias ALFIN y una canción esperanzada, 2012), voz plural de una misma esperanza;

- o el que publicara la revista RUSC sobre las competencias informacionales y digitales en la Educación Superior (2010), el estadio educativo donde se concentra la vanguardia en la generación del conocimiento y la experimentación del aprendizaje.

Que unos se dediquen al ámbito universitario y otros no, no significa sino que están segmentados; esto es, adaptados a distintas necesidades, porque de lo que se trata -como hemos dicho- es de resolver problemas, de contar con todos los implicados en cada ámbito (los que necesitan saber y los que saben, que a su vez deben aprender a enseñar; además de a colaborar para enseñar y aprender), con adecuar los medios y los contenidos a los contextos y situaciones. Unos contextos por los que iremos transcurriendo a lo largo de nuestra vida: el escolar, el ciudadano (que engloba, o debería, a todos los demás), el profesional, el universitario, el investigador, el creador, el artístico...

Puede entenderse de otra manera el fin de la ALFIN; su final como elemento definidor. Es una denominación cuestionada, apenas nacida, víctima de la misma vorágine ante la que intenta ser punto de referencia, brújula. La profusión de términos diferentes y alternativos utilizados se puede apreciar en el trabajo de Nieves González. Tiene su importancia, aunque es nimia ${ }^{2}$, si consideramos que lo importante son los contenidos que ese concepto debe abarcar. El problema muchas veces consiste en que los teóricos se dedican a discutir sobre los términos y sus posibles contenidos y no cruzan la línea de la definición para adentrarse en construir con los contenidos, planificando y programando esquemas prácticos, acciones, actividades, marcos, modelos... en definitiva, construyendo herramientas adecuadas.

2 Salvo que el cambio constante de denominación sea un elemento más en contra de su principal cometido (alumbrar la oscura infoxicación) o acabe desterrando lo que realmente es importante (la información) en beneficio del mero soporte.

Rev. Esp. Doc. Cient., N. ${ }^{\circ}$ Monográfico, 9-16, 2012. ISSN: 0210-0614. doi: 10.3989/redc.2012.mono.975 
Además le afecta la influencia de la lengua dominante en el escenario cambiante del conocimiento, la inglesa, que está por detrás del concepto y de las connotaciones de la ALFIN y de buena parte de las denominaciones alternativas. También a veces responde a situaciones simplemente circunstanciales; como seguramente sucede con el recién acuñado concepto de MIL (Media and Information Literacy) que obedece en buena medida a la fusión de dos grupos diferentes en el seno de la $\mathrm{UNESCO}^{3}$. Seguramente el dedicado a los Media influyera en la propuesta de la Unión Europea de finales de 2008 de incluir la "educación mediática" en los currículos obligatorios; una evolución de lo que hace dos décadas era "educación audiovisual" como mencionan Manuel Area y Amador Guarro. Independientemente de las razones, insistimos, demos importancia a las competencias que conforman su contenido, tal como se manifiesta en el trabajo de Nieves González, consideremos la conveniencia de dotarlas de autenticidad como subrayan Monereo y Badia y de servir para el aprendizaje competente que defienden Area y Guarro.

Un ejemplo de esta línea fluctuante en las denominaciones, e incluso en el ámbito que abarca la alfabetización informacional (el contenido de su discurso) se refleja claramente en este monográfico. No hemos indicado a ninguno de los autores cuál es el concepto de ALFIN que debían considerar y todos han respondido de una manera diferente; aunque en el fondo similar. Desde el concepto de competencia informacional más "restringido" (con sus inevitables incursiones en otros campos supuestamente diferentes) de Monereo y Badia, hasta la miríada que nos presenta Nieves González conteniendo todos los ámbitos de la información, pasando por la reivindicación de lo puramente informacional frente a lo tecnológico (aunque incluyéndolo, lógicamente) por parte de Glòria Durban y Ana Blasco seguramente influidas porque en el ámbito de la educación escolar es donde más se ha potenciado la inclusión de las nuevas herramientas TIC esperando que ellas solas lograran una revolución, sin analizar y programar adecuadamente objetivos no ilusorios, inversiones, formación o el propio soporte tecnológico que asegure conexiones adecuadas, el mantenimiento de equipos e infraestructuras, evite la obsolescencia y demás circunstancias relacionadas.

Estamos adelantando parte de lo que se recoge a continuación. ¿Qué podemos esperar?

Cuando nos propusieron coordinar este monográfico rápidamente nos planteamos su posible estructura. Nos rebelamos ante la idea clásica de subdividir el proceso en etapas y adjudicar cada una de ellas a un actor. Por otra parte, éramos conscientes de la necesaria interdisciplinariedad, de la diversidad de actores implicados y de los distintos escenarios desde los que se podría contemplar.

3 Cuando estas letras vean la luz habrá tenido lugar recientemente un congreso organizado por la UNESCO y la Sección de ALFIN de la IFLA para tratar el concepto y su desarrollo. Ya hubo en el Congreso de la IFLA del año pasado una reunión para ayudar a la UNESCO a redactar el documento base. 
Por eso la selección recayó en profesionales de distintos ámbitos, con funciones diferentes en ellos, tratando la misma cuestión desde puntos de vista diversos.

Tal vez se aprecie una mayor insistencia en los fundamentos, relacionados directamente con el aspecto didáctico y del aprendizaje "en el ámbito escolar". Aunque fuera así, es el ámbito clave ya que se sitúa en el momento en el que nos estamos construyendo como personas, diseñando nuestra identidad y conformando nuestra manera de ver el mundo: nuestra relación con él dependerá de cómo hayamos surgido de ese momento. Si vamos a vivir en la Sociedad de la Información (o del desconocimiento: Brey y otros, 2009) necesitamos contar con fundamentos sólidos sobre "esa sustancia integral de nuestro hoy".

Nieves González nos habla de una información que ha cambiado en sus fundamentos el propio ciclo de la comunicación y cómo se entienden sus protagonistas, lo que convierte en necesario que se modifiquen los contenidos y la forma de impartirlos/compartirlos. De ahí la profusión de esquemas y denominaciones distintos, algunos de los cuáles son mencionados. Así como experiencias reales: CI2, Plan Alba, etcétera.

Manuel Area y Amador Guarro también consideran que hay que redefinir el concepto de alfabetización para abarcar las multialfabetizaciones necesarias en el siglo actual (que es también el de la formación). Se centran en los conceptos de competencia y aprendizaje competente así como en las estrategias de enseñanza acordes, en las que la contextualización se convierte en esencial.

Carles Monereo y Antoni Badia partiendo de la idea de competencia se preguntan cuáles son los principales problemas a los que se enfrenta quien aprende y quien enseña, desde la perspectiva de cómo se aprende, adecuándolo a la competencia informacional, contemplándolo en un escenario de autenticidad, entendida como el planteamiento y resolución de problemas y situaciones reales.

Glòria Durban y Anna Blasco optan por presentar un modelo propio, en desarrollo, que ha evolucionado de esquemas previos. Nos muestran algunos de los elementos utilizados en cada una de las etapas. Con el valor añadido de confrontarlo con las competencias establecidas en la enseñanza obligatoria, en cuya consecución la biblioteca escolar tiene un papel que jugar.

Alejandro Uribe y María Pinto se acercan a la ALFIN en las bibliotecas públicas, el ámbito seguramente más complicado, ya que estas bibliotecas serían las encargadas de adecuarla al aprendizaje a lo largo de la vida, destinado a todo tipo de usuarios, e incluso de solventar posibles lagunas existentes en otro tipo de bibliotecas, como las escolares, que no tienen el desarrollo que pudiéramos desear.

Carmen Julia Hernández y José Erbez nos explican cuál ha sido su experiencia en una biblioteca universitaria: la implantación de su modelo, con el necesario convencimiento de autoridades y profesores, su evolución temporal, así como la del personal bibliotecario... Y, sobre todo, como lo han compartido, mediante convenio con otras universidades, además de su disposición a contar con nuevos protagonistas que quieran añadir su esfuerzo al proyecto además de beneficiarse de él. 
Por diferentes circunstancias han quedado fuera -cuando no existía tiempo material para encontrar alternativas- algunos colaboradores que formaban parte del plan inicial, hablantes de otra lengua, cuyos cometidos se centraban, por un lado en mostrarnos el papel de las organizaciones y las asociaciones, como las que fueron pioneras en el establecimiento de normas (ALA ${ }^{4}$, CAUL, ANZIIL), las que velan por los aspectos culturales como la UNESCO o incluso las que surgidas más tarde son activas en sus respectivos ámbitos (CILIP, SCONUL...) o tienen como objetivo ámbitos geográficos más amplios (IFLA). Su análisis habría ido acompañado de la esencia de cuestiones como la planificación, el marketing o el liderazgo, esenciales para conseguir los mejores resultados, una necesaria visibilidad, la expansión de los fundamentos y el apoyo de los proyectos y su ejecución.

Un último capítulo estaba dedicado exclusivamente a dos cuestiones: el perfil del profesional formador y el futuro de la ALFIN. Su ausencia, aunque importante, está matizada por el hecho de que algunos de los autores del monográfico, mencionan el primer aspecto. A veces olvidamos algo tan obvio como que en todas las actividades en las que intervienen las personas, éstas son lo fundamental, responsables en buena medida del éxito o del fracaso. En nuestro caso, los profesionales tienen que responder a unas necesidades muy específicas y diversas al mismo tiempo: no basta con el conocimiento de las materias susceptibles de ser enseñadas, ni con la destreza con las herramientas en las que se sustentan o que permiten acceder a ellas, ni del conocimiento de factores «externos" relacionados con ambos, contenidos y soportes; sino que deben reunir la capacidad necesaria para programar una formación, para impartirla, para adecuarse a perfiles diferentes, para comunicar y ser comunicado, para ser sujeto y objeto, para compartir, para trabajar en equipo, con todas sus implicaciones, para ser consciente de que es necesario aprender continuamente y del lugar y momento en el que vivimos, con sus luces y sus sombras... Es tan importante, que el propio Comité Permanente de la Sección de ALFIN de la IFLA ha creado un grupo de trabajo para intentar definir dichos perfiles, contemplando que los escenarios diferentes seguramente requieren competencias distintas.

Y también se ha abordado el futuro de la ALFIN, ya que era uno de los requisitos solicitados a cada uno de los autores y al que han respondido con diferente profundidad y extensión. Es difícil, además de aventurado, hacer alguna predicción en un ambiente cambiante, en el que hemos visto que hasta los términos están en discusión; aunque las predicciones no necesariamente busquen acertar el futuro sino más bien orientar hacia él. Por otra parte cuando se defiende una visión concreta de una disciplina se está implícitamente abordando su futuro, o al menos el futuro esperable. Cuando se hace un llamamiento a que

${ }^{4}$ La ALA, la asociación de bibliotecarios más grande del mundo cuenta con varias divisiones, entre ellas la ACRL, pionera en normas de ALFIN o la AASL, cuyas normas para bibliotecas escolares revisadas totalmente en 2007 son excelentes (AASL, 2007). 
se haga esto o aquello o que sería necesario conseguir esto otro, lo que se está diciendo es que debería existir un futuro de esa manera... ¿esperanzada, como en el monográfico de Alfared? En cualquier caso, nuestros autores lo han hecho adecuándolo al contexto en el que les habíamos comprometido.

No nos queda entonces sino agradecer la confianza depositada para llevar adelante esta empresa, la inestimable labor de quienes han intervenido en ella; así como la no tan agradable de los que han ayudado en todo el proceso de evaluación, a quienes no podemos mencionar por razones obviamente "ciegas".

Ahora es el momento -independientemente del formato y del contexto- de leer, disfrutar, reflexionar, copiar, anotar, glosar, obviar, repasar, reutilizar, mezclar, discutir, recelar, contraponer, discrepar, argumentar, valorar, pensar, enseñar, analizar, criticar, aborrecer, marcar, completar, enriquecer, emular, contrastar, matizar, destacar, redimensionar, reajustar, subrayar, echar por tierra, olvidar, replicar, interiorizar, adaptar, difundir, resumir, ignorar, repensar, reseñar, resaltar, citar, vilipendiar, releer, tuitear o twittear, comentar, compartir (con las preposiciones que sean necesarias)... el resultado; es decir, de hacer todo aquello que se hace con la información.

\section{Bibliografia}

AASL: American Association of School Libraries (2007). Standards for the 21 $1^{\text {st }}$ Century Learners. [Recuperado el 1 de mayo de 2012 de]: http://www.ala.org/aasl/guidelinesandstandards/learningstandards/standards

Alfared (2008): Foro Red Alfabetización informacional. Portal mantenido por la Secretaría de Estado de Cultura. [Recuperado el 30 de abril de 2012 de]: http://www.alfared.org/

Area, M.; Pessoa, T. (2012). De lo sólido a lo líquido: las nuevas alfabetizaciones ante los cambios culturales de la web 2.0. Comunicar: revista científica de comunicación y educación. DOI:10.3916/C38-2011-02-01.

Brey, Antoni; Innerarity, Daniel; Mayos, Gonçal (2009). La sociedad de la ignorancia. Barcelona: Zero Factory. 206 p. Proyecto de La Segunda Edad Contemporánea. ISBN 978-84-613-2970-0. [Recuperado el 1 de mayo de 2012 de]: http://www.infonomia.com/ pdf/sociedad_de_la_ignorancia_es.pdf

CEIP San Juan de la Peña (2011). Proyectos Documentales Integrados: recopilación de materiales de Rosa Piquín. [Recuperado el 1 de mayo de 2012 de]: http://cpsjphue. educa.aragon.es/proyectos-documentales-integrados

CI2 (2011): Competencias informáticas e informacionales. Portal de la Comisión mixta intersectorial CRUE-TIC y REBIUN. [Recuperado el 30 de abril de 2012 de]: http:// ci2.innn.es/

Competencias informacionales y digitales en la Educación Superior (2010): monográfico coordinado por Manuel Area Moreira. RUSC: Revista de Universidad y Sociedad del Conocimiento, v. 7, n. 2. [Recuperado el 1 de mayo de 2012 de]: http://rusc.uoc. edu/ojs/index.php/rusc/article/viewFile/v7n2-area/v7n2-competencias-informacionales-y-digitales-en-educacion-superior 
Fernández García, J. J. (2008). Más allá de Google. Barcelona: Zero Factory. 206 p. Proyecto de Infonomía.com. ISBN 978-84-612-1535-5. [Recuperado el 1 de mayo de 2012 de]: http://www.infonomia.com/pdf/Mas_alla_de_Google_2008.pdf

Gómez-Hernández, J.A. (2012). Alfabetización y autocrítica del comportamiento informacional mediante el humor gráfico de El Roto y Mauro Entrialgo. Notas ThinkEPI, 9 de enero. Anuario ThinkEPI. [Recuperado el 30 de abril de 2012 de]: http://www. thinkepi.net/alfabetizacion-y-autocritica-del-comportamiento-informacional-medianteel-humor-grafico-de-el-roto-y-mauro-entrialgo

Lara, T. (2010). Programa del módulo de competencias digitales UNIA-EOI 2010. Tíscar. com, 8 de abril de 2010. [Recuperado el 2 de mayo de 2012]: http://tiscar.com/2010/04/ 08/programa-del-modulo-de-competencias-digitales-unia-eoi-2010/

Otte, M. (2010). El crash de la información: los mecanismos de la desinformación cotidiana. Barcelona: Planeta. 347 p. ISBN 978-84-344-6923-5.

Ramonet, I. (2011). De resistencia y de ira: quince años. Le Monde diplomatique en español. 206 p. ISBN 978-84-95798-15-2.

Veintitantas experiencias ALFIN y una canción esperanzada (2012): monográfico coordinado por Felicidad Campal. 27 breves artículos. [Recuperado el 1 de mayo de 2012 de]: http://www.alfared.org/content/veintitantas-experiencias-alfin-y-una-canci-n-esperanzada/1287 